\title{
Estudo comparativo de testes diaǵnósticos para olho seco entre crianças saudáveis e portadoras de artrite reumatóidejuvenil
}

\author{
Comparative study of diagnostic tests for dry eye disease between healthy and juvenile \\ rheumatoid arthritischildren
}

\author{
Jayter Silva de Paula ${ }^{1}$ \\ Marco Antônio Bonini-Filho ${ }^{2}$ \\ Tarciso Schirmbeck ${ }^{3}$ \\ Virginia Paes Leme Ferriani ${ }^{4}$ \\ Maria de Lourdes Veronese Rodrig'ues ${ }^{5}$ \\ Erasmo Romão
}

Trabalho realizado no Ambulatório de Oftalmologia do Hospital das Clínicas da Faculdade de Medicina de Ribeirão Preto, Universidade de São Paulo/HCFMRPUSP.

Médico Assistente-Doutor do Setor de Uveítes, Departamento de Oftalmologia, Otorrinolaringologia e Cirurgia de Cabeça e Pescoço da Faculdade de Medicina de Ribeirão Preto da Universidade de São Paulo - USP.

${ }^{2}$ Médico Residente do Serviço de Oftalmologia, Departamento de Oftalmologia, Otorrinolaringologia e Cirurgia de Cabeça e Pescoço da Faculdade de Medicina de Ribeirão Preto da Universidade de São Paulo - USP.

Pós-graduando, nível doutorado, do Departamento de Oftalmologia, Otorrinolaringologia e Cirurgia de Cabeça e Pescoço da Faculdade de Medicina de Ribeirão Preto da Universidade de São Paulo - USP.

${ }^{4}$ Professora Doutora do Departamento de Puericultura e Pediatria da Faculdade de Medicina de Ribeirão Preto da Universidade de São Paulo - USP.

Professores Associados do Departamento de Oftalmologia, Otorrinolaringologia e Cirurgia de Cabeça e Pescoço da Faculdade de Medicina de Ribeirão Preto da Universidade de São Paulo - USP

Endereço para correspondência: Jayter Silva de Paula Departamento Oftalmologia, Otorrinolaringologia e Cirurgia de Cabeça e Pescoço, Av. Bandeirantes, $\mathrm{n}^{\mathrm{a}} 3900$, $12^{\underline{q}}$ andar - Ribeirão Preto (SP) - CEP 14049-900

E-mail: jayterdepaula@msn.com

Recebido para publicação em 04.12.2003

Versão revisada recebida em 27.04.2004

Aprovação em 12.05.2004

\section{RESUMO}

Objetivo: Comparar achados diagnósticos de olho seco em crianças normais e com artrite reumatóide juvenil. Métodos: Neste estudo transversal, 30 olhos de 15 pacientes com artrite reumatóide juvenil (grupo 1) e 22 olhos de 11 crianças-controle (grupo 2) foram examinados clinicamente e submetidos a testes para ceratoconjuntivite seca: Schirmer tipo 1, tempo de quebra do filme lacrimal e coloração com rosa bengala. Resultados: Seis crianças com artrite reumatóide juvenil apresentaram um ou mais sintomas de ceratoconjuntivite seca $(40 \%)$ e cinco destas $(83,3 \%)$ mostravam meibomite ou outros sinais dessa afecção. Nenhuma criança do grupo 2 apresentou sinais ou sintomas de ceratoconjuntivite seca. No teste de Schirmer não se observou diferença significativa entre os grupos 1 e 2 $(\mathrm{p}=0,156)$. Entretanto, o tempo de quebra do filme lacrimal foi significativamente menor no grupo $1(\mathrm{p}=0,0005)$ e de maneira semelhante, o escore do teste de rosa bengala foi significativamente maior no grupo $1(\mathrm{p}=0,0038)$. Cinco das 15 crianças estudadas do grupo 1 apresentaram um ou mais testes alterados e tiveram diagnóstico definitivo de ceratoconjuntivite seca, ao passo que quatro $(26 \%)$ tiveram o diagnóstico de provável ceratoconjuntivite seca. No grupo 2, nenhuma criança apresentou mais de um teste positivo. Conclusões: Sinais e sintomas de ceratoconjuntivite seca constituem achados comuns em crianças com artrite reumatóide juvenil. Embora apenas o tempo de quebra do filme lacrimal e a marcação com rosa bengala tenham tido diferença significativa entre os grupos, parece haver tendência a resultados piores nos testes de olho seco realizados em crianças com artrite reumatóide juvenil.

Descritores: Artrite reumatóide juvenil/diagnóstico; Ceratoconjuntivite seca/diagnóstico; Técnicas de diagnóstico oftalmológico; Lágrimas/análise; Rosa bengala/uso diagnóstico; Criança; Estudo comparativo

\section{INTRODUÇÃO}

A instabilidade do filme lacrimal está associada a inúmeras doenças oculares e decorre de alterações do volume, da composição e da hidrodinâmica da lágrima ${ }^{(1)}$. O olho seco, designação freqüente dessa instabilidade, é uma das entidades nosológicas mais freqüentes na prática oftalmológica em todo o mundo, sendo a irritação, sensação de corpo estranho e ardência ocular os sintomas mais comuns ${ }^{(2)}$.

$\mathrm{O}$ diagnóstico de ceratoconjuntivite seca $(\mathrm{KCS})$ pode ser estabelecido na evidência de achados de olho seco com a positividade de alguns testes 
específicos para tal ${ }^{(1)}$. À parte da KCS idiopática, algumas afecções transcorrem freqüentemente com olho seco, tais como: artrite reumatóide, síndrome de Sjögren e condições locais causadas por trauma, infecções ou drogas ${ }^{(3)}$.

A KCS é considerada a complicação mais comum em pacientes adultos com artrite reumatóide, sendo, porém, pouco estudada em pacientes com artrite reumatóide juvenil (ARJ) ${ }^{(4-5)}$. Torna-se interessante, então, o estudo comparativo, de testes diagnósticos para olho seco, entre pacientes com ARJ e crianças normais.

\section{MÉTODOS}

Foi realizado um estudo transversal, com 30 olhos de 15 pacientes com diagnóstico de qualquer forma de ARJ (grupo 1) e 22 olhos de 11 crianças sem ARJ e patologias oculares, tidas como controle (grupo 2). Os diagnósticos de ARJ foram dados seguindo os critérios do Colégio Americano de Reumatologia ${ }^{(6)}$.

Após consentimento dos responsáveis e explicação pormenorizada do presente estudo, uma avaliação clínica inicial foi realizada, com anamnese e exame oftalmológico completo, junto ao Setor de Uveítes do Ambulatório de Oftalmologia do Hospital das Clínicas da Faculdade de Medicina de Ribeirão Preto (USP), no período de fevereiro a agosto de 2002.

A anamnese foi dirigida a queixas de: dor e ardor ocular, lacrimejamento, visão borrada, fotofobia, sensação de corpo estranho ou de secura ocular, peso nas pálpebras e hiperemia conjuntival $^{(7)}$. Considerou-se, ainda, a presença de atividade e tratamento da artrite reumatóide juvenil, assim como a administração de medicações de uso sistêmico ou ocular.

Realizou-se o exame de lâmpada de fenda, com atenção especial à superfície ocular, composição e tamanho do menisco lacrimal, alterações de margem palpebral, conjuntiva e córnea, além da procura objetiva de sinais clássicos de uveíte anterior. $\mathrm{O}$ filme lacrimal foi estudado usando-se os seguintes testes: Schirmer tipo 1 (TS), tempo de quebra do filme lacrimal (TQFL) e marcação com o corante rosa bengala (RB).

Inicialmente, pesquisou-se o TQFL com a instilação de uma gota de fluoresceína sódica $0,25 \%$ e observação do paciente à lâmpada de fenda com os olhos abertos, a fim de medir o tempo para ocorrência do primeiro ponto de quebra do filme lacrimal na córnea, com luz de cobalto, em ambos os olhos. Valores menores de 10 segundos foram considerados positivos como indicativo de olho $\mathrm{seco}^{(8)}$.

Posteriormente, realizou-se o TS com a colocação de papel de filtro padrão milimetrado em fundo de saco conjuntival inferior de ambos os olhos, por 5 minutos. Medidas menores que $10 \mathrm{~mm}$ foram considerados como positivos ${ }^{(9)}$.

Por último, instilou-se 1 gota de colírio de rosa bengala $1 \%$ em região de fórnice inferior de ambos olhos. Em seguida observou-se, com luz anerita, a localização e intensidade de coloração da córnea e das conjuntivas nasal e temporal. A impregnação dessas três áreas foi quantificada por uma escala numérica em cada região, variando de 0-3 (escala de Bijsterved), na qual: 0 significa ausência de coloração, 1 coloração puntiforme, 2 coloração com pontos confluentes e 3 coloração de placas extensas. Pontuação na escala maior ou igual a 3 foi considerado como positivo ${ }^{(10)}$. Crianças com positividade em 2 ou mais testes tiveram o diagnóstico definitivo de KCS. Tiveram o diagnóstico de provável KCS, aquelas com positividade em apenas 1 teste.

Todos os pacientes com ARJ (grupo 1) tiveram além do descrito, o exame de rotina do Setor de Uveítes para outros achados oftalmológicos pertinentes à doença de base. Além disso, os indivíduos que apresentaram quadro oftalmológico de olho seco foram tratados com colírios lubrificantes.

A análise estatística foi realizada comparando-se os grupos através de média, desvio-padrão e do teste não-paramétrico U Mann Whitney, com significância para $\mathrm{p}<0,05$.

\section{RESULTADOS}

As idades das crianças variaram de 6 a 15 anos no grupo 1 $(9,9 \pm 3,1$ anos) e de 5 a 14 anos no grupo 2 (7,9 $9 \pm 2,7$ anos). A proporção masculina:feminina foi de 1: 2 no grupo 1 e de 1,75:1 no grupo 2 .

Nenhuma criança fazia uso de medicações de uso ocular, sendo que todas crianças do grupo 1 utilizavam alguma medicação sistêmica (Tabela 1). Todas as crianças do grupo 1 se apresentavam sem atividade da ARJ, com exceção do caso número 5 que apresentava inchaço indolor em ambos os joelhos, no período do estudo. Além disso, não foi constatada presença de sinais de uveíte aguda ou crônica em nenhuma criança desse grupo.

Seis crianças com ARJ (grupo 1) apresentaram um ou mais sintomas de KCS $(40 \%)$ e cinco destas $(83,3 \%)$ mostravam meibomite ou outros sinais de KCS, como o decréscimo do menisco lacrimal. Nenhuma criança do grupo 2 apresentou sinais ou sintomas de KCS.

No TS, não se observou diferença significativa entre os grupos 1 e 2 (respectivamente, 18,33 $\pm 6,20 \mathrm{~mm}$ e 20,63 $\pm 3,81 \mathrm{~mm}$, $\mathrm{p}=0,156$ ), sendo constatada positividade para apenas 4 olhos (13\%) do grupo 1. Entretanto, o TQFL foi significativamente menor no grupo $1(7,73 \pm 3,97 \mathrm{~s}$ contra $10,31 \pm 1,88 \mathrm{~s}, \mathrm{p}=0,0005)$, sendo considerado positivo em 23 olhos do grupo 1 (77\%) contra 7 olhos do grupo 2 (32\%). Da maneira semelhante, o escore do teste de RB foi significativamente maior no grupo 1 $(1,33 \pm 1,49$ contra $0,40 \pm 0,50, p=0,0038)$, sendo, no entanto, considerado positivo em apenas 6 olhos (20\%) do grupo 1 .

Das 15 crianças estudadas, cinco (33\%) tiveram diagnóstico definitivo de KCS e quatro (26\%) tiveram o diagnóstico dito provável. No entanto, apenas três (20\%) crianças tiveram positividade para mais de dois testes em ambos olhos (tabela 1). No grupo 2, nenhuma criança apresentou mais que um teste positivo, dentre os estudados. 


\begin{tabular}{|c|c|c|c|c|c|c|c|c|}
\hline Paciente & $\begin{array}{l}\text { Idade } \\
\text { (anos) }\end{array}$ & Sexo & $\begin{array}{l}\text { Forma de } \\
\text { ARJ }\end{array}$ & $\begin{array}{l}\text { Medicação } \\
\text { em uso }\end{array}$ & $\begin{array}{c}\text { TS } \\
\text { OD, OE }\end{array}$ & $\begin{array}{l}\text { TQFL } \\
\text { OD, OE }\end{array}$ & $\begin{array}{c}\text { RB } \\
\text { OD, OE }\end{array}$ & $\begin{array}{l}\text { Resultado } \\
\text { (KCS) }\end{array}$ \\
\hline 1 & 15 & $\mathrm{~F}$ & Poliarticular & $\begin{array}{l}\text { Ranit., naprox., } \\
\text { ac. fólico, MTX }\end{array}$ &,-- &,++ &,++ & Presente \\
\hline 2 & 11 & $\mathrm{~F}$ & Sistêmico & $\begin{array}{l}\text { Ranit, ibup., ac. fólico, } \\
\text { MTX, HCLQ, pred. }\end{array}$ &,+- &,++ &,-- & Presente \\
\hline 3 & 9 & $\mathrm{~F}$ & Poliarticular & Cimet., ac. fólico, MTX &,-- &,++ &,-- & Provável \\
\hline 4 & 15 & $\mathrm{~F}$ & Poliarticular & $\begin{array}{l}\text { MTX, HCLQ, AZA, } \\
\text { ac. fólico }\end{array}$ &,-- &,++ &,-- & Provável \\
\hline 5 & 11 & M & Poliarticular & Cimet., pirox., MTX &,+- &,++ &,++ & Presente \\
\hline 6 & 6 & $\mathrm{~F}$ & Poliarticular & Pirox., ac. fólico, MTX &,-- &,++ &,-- & Provável \\
\hline 7 & 7 & M & Pauciarticular & Pirox. &,-- &,++ &,-- & Provável \\
\hline 8 & 9 & $\mathrm{~F}$ & Pauciarticular & Pirox. &,-- &,++ &,-+ & Presente \\
\hline 9 & 9 & M & Poliarticular & Pirox., MTX, ac. fólico &,++ &,++ &,-+ & Presente \\
\hline
\end{tabular}

\section{DISCUSSÃO}

A ARJ atualmente é considerada como uma doença sistêmica e não somente como uma condição inflamatória de articulações. Os principais locais de manifestação extra-articular dessa entidade são os olhos, rins e coração ${ }^{(11)}$.

A inflamação intra-ocular, iridociclite, é usualmente assintomática, crônica, não granulomatosa e bilateral. Tem maior predileção por crianças do sexo feminino, com ARJ do tipo pauciarticular, de menor idade e com exames positivos para anticorpos antinúcleo ${ }^{(11)}$. Em crianças com ARJ, a iridociclite assintomática é a apresentação ocular mais comum, sendo a KCS um achado ocular $\operatorname{raro}^{(5,12)}$.

No presente estudo, todos os testes diagnósticos para KCS apresentaram-se com relativa maior positividade no grupo 1 (pacientes com ARJ), no entanto, como mencionado anteriormente, apenas o TQFL e a marcação com RB apresentaram diferenças estatisticamente significativas entre os dois grupos. Esse fato poderia contradizer achados anteriores acerca da não associação direta da $\mathrm{ARJ}$ com $\mathrm{KCS}^{(5,12)}$. Porém, em um estudo mais recente ${ }^{(4)}$, os autores observaram uma prevalência $12 \%$ de KCS em pacientes com ARJ, valor próximo dos $20 \%$ encontrado no presente estudo. Nos resultados obtidos, encontramos uma maior positividade para KCS em crianças do sexo feminino, ao contrário daquele estudo, no qual houve predominância de KCS em indivíduos do sexo masculino. Esse fato poderia ser explicado, como o próprio autor discute, pela maior proporção de atendimento de meninos no local, decorrente de questões culturais daquele país (Índia).

A diferença significativa entre os grupos apenas na comparação entre o TQFL e a marcação com RB se justificaria pela possibilidade de que, em crianças com ARJ, o quadro de KCS seria menos intenso, com pouca relação com a produção aquosa lacrimal (valores de TS altos). Um achado, a meibomite, poderia explicar algumas das alterações observadas, apesar do número de crianças estudadas ainda ser pequeno.
Deve-se acrescentar que todas as crianças do grupo 1, com testes diagnósticos positivos, estavam em uso de alguma medicação sistêmica. No entanto, apesar dos sabidos efeitos colaterais dessas drogas no olho, a sua frequiência é baixa e a sua intensidade geralmente é mínima, principalmente se considerarmos que as doses utilizadas são, também, usualmente baixas $^{(13-14)}$. De forma semelhante, não se conseguiu correlacionar a atividade da doença com intensidade de alterações lacrimais, haja vista que apenas uma criança do grupo 1 apresentava sinais de atividade de ARJ.

\section{CONCLUSÃO}

Com o exposto anteriormente, além do fato de que foram mais comuns as queixas de olho seco no grupo 1, pode-se dizer que existe uma tendência a alterações do filme lacrimal em crianças com ARJ. No entanto, um estudo complementar, com um número maior de indivíduos estudados e uma análise multivariada correlacionando outros fatores, tais como positividade para anticorpos antinúcleo e velocidade de hemossedimentação, tempo de diagnóstico e subtipo de ARJ, dose dos medicamentos sistêmicos utilizados, dentre outros, se faz necessário para se estabelecer definitivamente os mecanismos envolvidos nas alterações do filme lacrimal observadas.

\section{ABSTRACT}

Purpose: To compare dry eye diagnostic findings in juvenile rheumatoid arthritis patients and normal children. Methods: For this transversal study, 30 eyes of 15 patients with juvenile rheumatoid arthritis (group 1) and 22 eyes of 11 normal controls (group 2) were examined clinically and underwent tests for keratoconjunctivitis sicca: Schirmer's 1, tear film break-up time and rose bengal staining tests. Results: Six children with juvenile rheumatoid arthritis presented one or 
more symptoms of keratoconjunctivitis sicca (40\%) and five of them $(83.3 \%)$ presented meibomitis or other signs of this disease. In group 2, no child presented symptoms or signs of keratoconjunctivitis sicca. Mean Schirmer test did not differ between group 1 and $2(\mathrm{p}=0.156)$. However, the mean tear film break-up time was significantly reduced in group $1(\mathrm{p}=0.0005)$ and the mean rose Bengal staining score in group 1 was significantly greater than in group $2(\mathrm{p}=0.0038)$. Five of the fifteen children of group $1(33 \%)$ have two or more abnormal tests and were diagnosed as having definite keratoconjunctivitis sicca, while four children $(26 \%)$ were labeled with probable keratoconjunctivitis sicca. No child of group 2 had more than one positive test. Conclusions: Signs and symptoms of keratoconjunctivitis sicca appear to be a common ocular finding in juvenile rheumatoid arthritis children. Although only tear film break-up time and rose bengal staining score were significantly different in these groups, there was a trend toward worsening of the other dry eye tests in juvenile rheumatoid arthritis children.

Keywords: Arthritis, juvenile rheumatoid/diagnosis; Keratoconjunctivitis sicca/diagnosis; Diagnosis techniques, ophthalmological; Tears/analysis; Rose bengal/diagnostic use; Children; Comparative study

\section{REFERÊNCIAS}

1. Murillo-Lopez F, Pflugfelder SC. Dry eye. In: Krachmer JH, Manis MJ, Holland EJ, editors. Cornea. St. Louis: Mosby; 1997. p.663-86.

2. Nishiwaki-Dantas MC. Atualização em olho seco. Arq Bras Oftalmol. 2000; 62(1):101-5.

3. Bron AJ. Diagnosis of dry eye. Surv Ophthalmol. 2001;45(Suppl 2):S221-6

4. Jain V, Singh S, Sharma A. Keratoconjuntivictis sicca is not uncommon in children with juvenile rheumatoid arthritis. Rheumatol Int. 2001;20(4):159-62.

5. Chylack LTJr, Bienfang DC, Bellows AR, Stillman JS. Ocular manifestations of juvenile rheumatoid arthritis. Am J Ophthalmol. 1975;79(6):1026-33.

6. Cassidy JT, Levinson JE, Bass JG, Baum J, Brewer EJ, Fink CW, et al. A study of classification criteria for diagnosis of juvenile rheumatoid arthritis. Arthritis Rheum. 1986;29(2):274-81.

7. Begley CG, Caffery B, Chalmers RL, Mitchell GL. Use of the dry eye questionnaire to measure symptoms of ocular irritation in patients with aqueous tear deficient dry eye. Cornea. 2002;21(7):664-70.

8. Gilbard JP. Dry eye disorders. In: Albert DM, Jakobiec F, editors. The principles and practice of ophthalmology: basic sciences. Philadelphia: WB Saunder; 1994. p.257-76.

9. Farris RL, Gilbard JP, Stuchell RN, Mandel ID. Diagnostic test in keratoconjunctivitis sicca. CLAO J. 1983;9(1):23-8.

10. Van Bijsterveld OP. Diagnostic tests in the sicca syndrome. Arch Ophthalmol. 1969;82(1):10-4.

11. Cassidy JT, Petty RE. Juvenile rheumatoid arthritis. In: Cassidy JT, Petty RE. editors. Textbook of pediatric rheumatology. Philadelphia: Saunders; 1995. p.133-233.

12. Kanski JJ. Uveitis in juvenile chronic arthritis: incidence, clinical features and prognosis. Eye. 1988;2(Pt 6):641-5.

13. Doroshow JH, Locker GY, Gaasterland DE, Hubbard SP, Young RC, Myers CE. Ocular irritation from high-dose methotrexate therapy: pharmacokinetics of drug in the tear film. Câncer. 1981;48(10):2158-62.

14. Fraunfelder FT, Meyer SM. Drug-induced ocular side effects and drug interactions. Philadelphia: Lea \& Febiger; 1982.

\section{Congresso da Sociedade Brasileira de Retina e Vítreo}

\section{8 a 21 de Março de 2005 Angra dos Reis - RJ}

\section{INFORMAC̣ÕES: LK Assessoria e Promoções}

Tel.: (21) 3778-2512

Fax: (21) 3878-1548

E-mail: evento@lk.com.br 